American Journal of Nanotechnology 1 (2): 62-67, 2010

ISSN 1949-0216

(C) 2010 Science Publications

\title{
Molecular Dynamics Investigation of Cutting Force in Nanometric Cutting of Monocrystalline Silicon
}

\author{
Y.H. Chen, F.Z. Fang, X.D. Zhang and X.T. Hu \\ State Key Laboratory of Precision Measuring Technology and Instruments, \\ Centre of Micron no Manufacturing Technology, \\ Tianjin University, Tianjin 300072, China
}

\begin{abstract}
Problem statement: Ultra-precision machining of monocrystalline silicon has become tremendously important in microelectronic, micromechanical and optical element manufacturing and the mechanism of nanometric cutting monocrystalline silicon has been a focused research topic. Molecular Dynamics (MD) simulation is developed as an effective way for studying the material removal process at nanometric scale. Approach: Molecular Dynamics (MD) simulation of nanometric cutting is conducted on monocrystalline silicon with three dimensional diamond tool of $0^{\circ}$ rake angle to investigate the cutting force. The cutting process is explicitly described by the combination of Morse and Tersoff potential. Nanometric cutting process is explained using the mechanism of tool extrusion induced lattice slip and distortion. Results: Cutting force vibration phenomenon is investigated in detail. Simulations on different tool cutting edges are carried out with various cutting depth to study the cutting force variation. The threshold cutting depth for chip formation is given. Conclusion: It is noted that cutting force vibration which is the main reason for tool wear and surface fracture is the result of tool extrusion and material deformation. The cutting force curves are distinct from the threshold cutting depth of chip formation and cutting forces decrease as the cutting edge value increase when cutting with same cutting depth.
\end{abstract}

Key words: Monocrystalline silicon, cutting force, Molecular Dynamics (MD), material removal process, cutting depth, threshold cutting, cutting tool, Symmetry Parameters (CSP), hydrostatic pressure

\section{INTRODUCTION}

Monocrystalline silicon is widely used in infrared optics and electronic applications which require optical quality surfaces and intricate features (De Chiffrea et al., 2003). Ultra-precision machining with diamond tools can make the production of components with nanometric surface finish and sub-micron level form accuracy possible (Fang and Venkatesh, 1998). In order to fabricate well-made components, it is necessary to study the mechanism of nanometric cutting monocrystalline silicon, especially the cutting force which plays an important role in tool wear, surface finish and form accuracy. However, as nanometric cutting involves changes in only a few nanometres from the surface, it is difficult to observe the machining process and measure the cutting force accurately. Molecular Dynamics (MD) simulation is developed as an effective way for studying the material removal process at nanometric scale (Evans, 1983).
In nanometric machining of monocrystalline materials, cutting force is predictable if the change in crystallographic orientation of the substrate material with respect to the cutting direction is known (Lee, 1990). However, little is known about the real reason for the cutting force vibration which is an important factor in tool wear and few studies were done on the chip formation mechanism from the view of cutting force.

In this study, the results of the MD simulations on the cutting forces in nanometric cutting of monocrystalline silicon are presented. The simulated cutting process was explained with the view of lattice slip and distortion and the reason for cutting force vibration was investigated in detail. Simulations on different tool cutting edges were carried out with various cutting depths and the threshold cutting depth for chip formation was also given.

Corresponding Author: Y.H. Chen, State Key Laboratory of Precision Measuring Technology and Instruments, Centre of Micron no Manufacturing Technology, Tianjin University, Tianjin 300072, China 


\section{MATERIALS AND METHODS}

MD 3D cutting model: The simulation model which consists of a 3D diamond tool and a monocrystalline silicon work piece is shown in Fig. 1. The work piece has been divided into three different layers, namely boundary, thermo layer and Newton layer. Boundary is used to fix the work piece and avoid unexpected movement of the work piece in cutting. Thermo layer maintains a constant temperature of $300 \mathrm{~K}$ for conducting the generated high heat away from the cutting region. Atoms in Newton layer interact with the tool follow Newton's law. The three dimensional cutting tool in the simulation is modelled with $0^{\circ}$ rake angle and a $12^{\circ}$ clearance angle.

MD simulation conditions: Details of the work piece and simulation conditions are summarized in Table 1. The work piece consists of 57600 atoms, while 4444 atoms of the tool. All the boundary conditions are set to be fixed for 3 dimensional simulations. The initial temperature of the work piece is $300 \mathrm{~K}$. The simulation time step is $1 \mathrm{fs}$.

Inter-atomic potential: There are three different atomic interactions in the MD simulations processes: (1) the interaction in the work piece; (2) the interaction between the work piece and the cutting tool; (3) the interaction in the tool.

In the simulations, Tersoff potentials are employed for the interaction among $\mathrm{Si}$ atoms in the work piece and Morse potential for the interaction between diamond tool and silicon. The tool is regarded as rigid body so that the atoms in the tool are fixed relatively to each other and with no interaction force among the tool atoms. The parameters used in the potential energy is shown in Table 2.

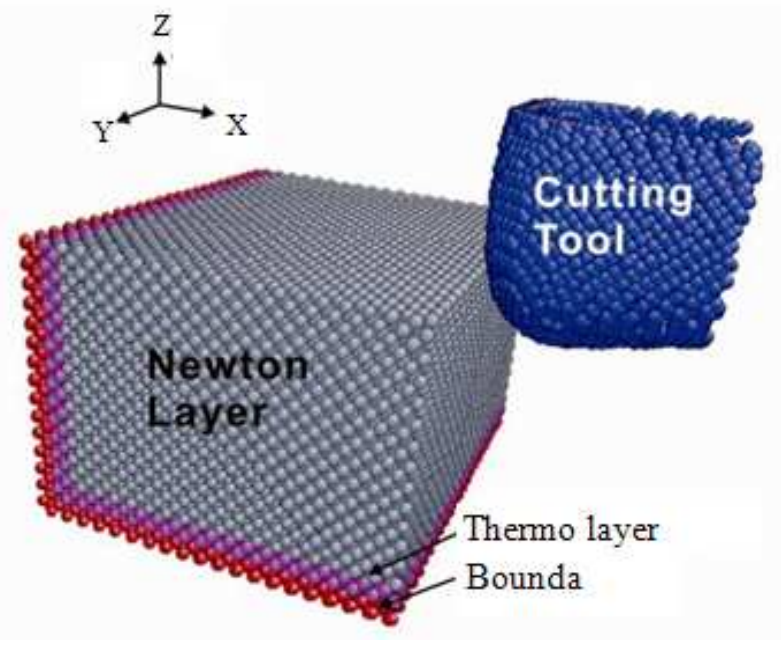

Fig. 1: Schematic MD simulation model

Table 1: Work piece and simulation conditions Work piece and simulation parameters

\begin{tabular}{|c|c|c|c|}
\hline Work material & Monocrystalline silicon & Tool edge radius & $1,1.5,2$ and $2.5 \mathrm{~nm}$ \\
\hline Material structure & Diamond cubic & Potential & Morse and Tersoff \\
\hline Work dimensions & $20 \mathrm{a} \times 15 \mathrm{a} \times 24 \mathrm{a}, \mathrm{a}=5.431 \AA$ & Cutting direction & $\langle 100\rangle$ on $(100)$ \\
\hline Tool dimensions & $4 \mathrm{~nm}$ tool radius & Cutting speed & $400 \mathrm{~ms}^{1}$ \\
\hline
\end{tabular}

Table 2: Potential parameters

Tersoff potential parameters

\begin{tabular}{|c|c|c|c|c|c|}
\hline $\mathrm{A}(\mathrm{eV})$ & $1.8308 \times 10^{\square}$ & $\beta$ & $1.1 \times 10^{\square}$ & $\mathrm{H}$ & $-5.9825 \times 10^{-1}$ \\
\hline $\mathrm{B}(\mathrm{eV})$ & $4.7118 \times 10^{\square}$ & $\mathrm{n}$ & $7.8734 \times 10^{-1}$ & $\mathrm{R}(\mathrm{nm})$ & 0.27 \\
\hline$\lambda\left(\mathrm{nm}^{1}\right)$ & 24.799 & $\mathrm{c}$ & $1.0039 \times 10^{5}$ & $\mathrm{~S}(\mathrm{~nm})$ & 0.30 \\
\hline$\mu\left(\mathrm{nm}^{1}\right)$ & 17.322 & $\mathrm{~d}$ & $1.6217 \times 10^{1}$ & & \\
\hline \multicolumn{6}{|c|}{ Morse potential parameters } \\
\hline $\mathrm{D}(\mathrm{eV})$ & $\alpha\left(\mathrm{nm}^{1}\right)$ & $\mathrm{r}_{0}(\mathrm{~nm})$ & & & \\
\hline 0.423 & 4.6487 & 1.9475 & & & \\
\hline
\end{tabular}




\section{RESULTS AND DISCUSSION}

Simulated nanometric cutting process: The nanometric cutting process was investigated based on MD simulations for the monocrystalline silicon work piece. The simulated cutting processes are illustrated in Fig. 2. There are obvious lattice slip and distortion during every time step of the tool and material interaction (Fig. 2a-c). It is shown that the effective rake angle, which is always negative (Fang et al., 2007), produces hydrostatic pressure, which has decisive effect on chip formation, subsurface deformation and machined surface generation.

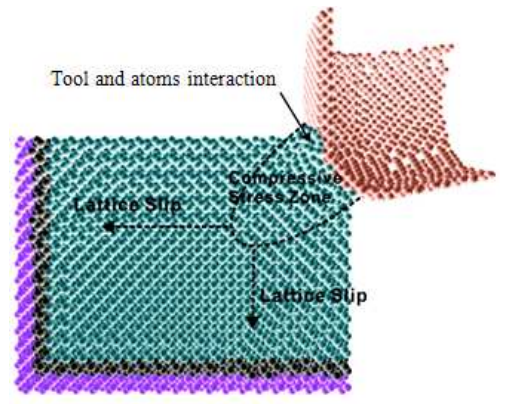

(a)

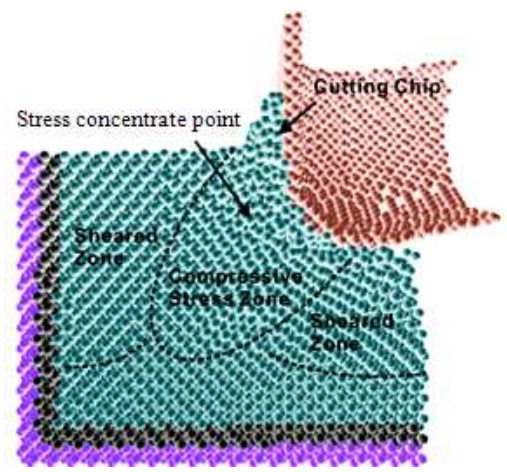

(b)

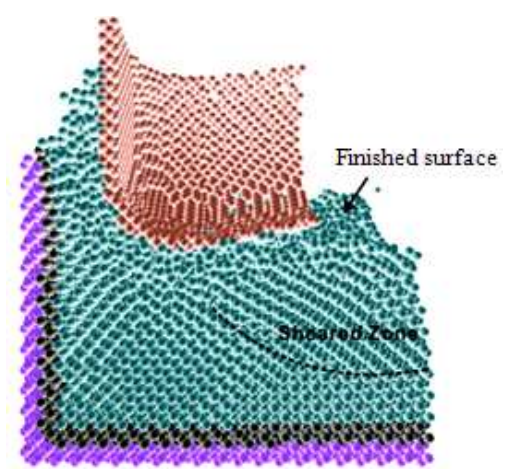

(c)

Fig. 2: Cross-sectional 3D cutting process: (a) Tool and material initial interaction; (b) Chip formation; (c) Machined surface
Figure 2a shows the initial interaction between the tool and the work piece. Cutting tool first introduce hydrostatic pressure underneath the tool edge. Due to the chip extrusion, lattice distortion comes into being in the contact region. Meanwhile, the compressive stress zone leads to equivalent scale lattice slip to the whole work piece. As the tool keeps on moving, compressive stress zone also moves forward, which is shown in Fi. 2(b). Stress concentrate point separates the deformed silicon atoms to flow to different directions and form cutting chips and machined surface respectively. The machined surface is generated after cutting, which is smooth and exhibits spring back because of the material relaxation, as shown in Fig. 2c.

Cutting force vibration: The force acting on an individual atom is obtained by summing the forces contributed by the surrounding atoms. The forces are calculated from the inter-atomic Morse potentials. Cutting force is expressed by the summation of the total force on all tool atoms.

Figure 3 shows the cutting force vibration in the cutting process. Figure $3 \mathrm{a}$ is the typical curves of tangential and normal cutting forces of $2 \mathrm{~nm}$ cutting depth with a $2 \mathrm{~nm}$ tool cutting edge. The cutting force vibration is accordance with the cutting process mentioned in Section 3.1. While Fig. 3b shows the frequency of the cutting force vibration. Two main frequencies, which represent small scale vibration and tapered sinusoidal oscillation, could be seen respectively.

Initially, the cutting forces are negative due to the attraction tool surface exert on the work piece atoms when cutting tool close to but have no contact with the work piece. When tool edge imposes hydrostatic pressure on the work piece, compressive stress is created and lattice slip is formed. Small vibration could be seen at the beginning. Cutting force increases gradually as the interface between tool and work piece aggrandizes until the tool face fully contacts with the material. Lattice disorder and atom flow at the adjacent region between the tool and the work piece is the main reason for the small vibration, as shown in Fig. 4.

Figure $4 \mathrm{a}$ is the schematic for lattice disorder using Centro Symmetry Parameters (CSP) (Tsuzuki et al., 2007). The CSP for each atom is defined as follows:

$\mathrm{P}=\sum_{\mathrm{i}=1, \mathrm{n}}\left|\mathrm{R}_{\mathrm{i}}+\mathrm{R}_{\mathrm{i}+\mathrm{n}}\right|^{2}$

where, $R_{i}$ and $R_{i+n}$ are the vectors corresponding to the nearest neighbors in the material lattice. In a centrosymmetric material, each atom has certain pairs of equal and opposite bonds among its nearest neighbors. As the material is distorted, these bonds will change direction and/or length. The CSP is zero for atoms in perfect lattice but are of different values for distortion. 


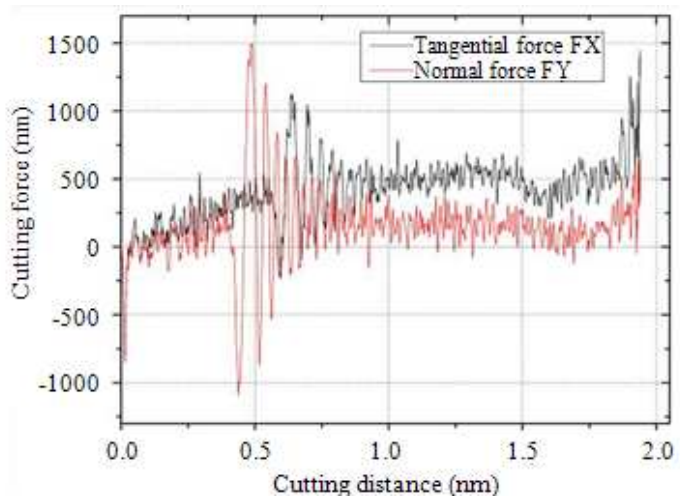

(a)

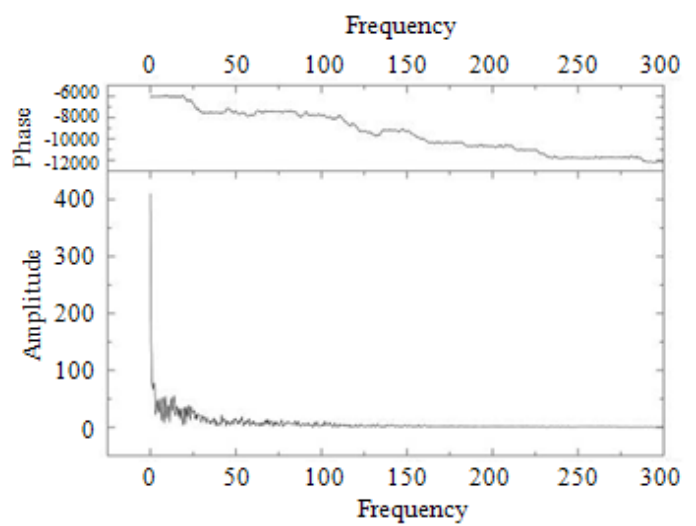

(b)

Fig. 3: Cutting force vibration: (a) Cutting force; (b) Frequency of the cutting force

The cutting chips are almost amorphous due to the tool extrusion induced lattice distortion and lattice slip, which can be seen in Fig. 4b, using Radial Distribution Function (RDF) measurements:

$\mathrm{g}\left(\mathrm{r}, \mathrm{r}^{\prime}\right)=\rho\left(\mathrm{r}, \mathrm{r}^{\prime}\right) / \rho(\mathrm{r})$

Where, $\rho\left(r, r^{\prime}\right)$ is the atom number in $r \rightarrow r+\Delta r, \rho(r)$ is the average ensemble parameter. RDF quantitative analyzes the structural changes of the work piece in histogram form by evaluating the statistical information of bond length variation. RDF results are in accordance with the laser micro-Raman spectroscopy of the real silicon cutting chip, as shown in Fig. 4c.

Tapered sinusoidal oscillation occurs when extrusion induced lattice slip extents to the threshold and the material is sheared at the lattice slip layer. The oscillation is the main reason for tool wear and surface fracture. Then, new balance is achieved after shearing and small oscillation takes place as the tool keeps on moving by the extrusion induced material deformation and finished surface generation.

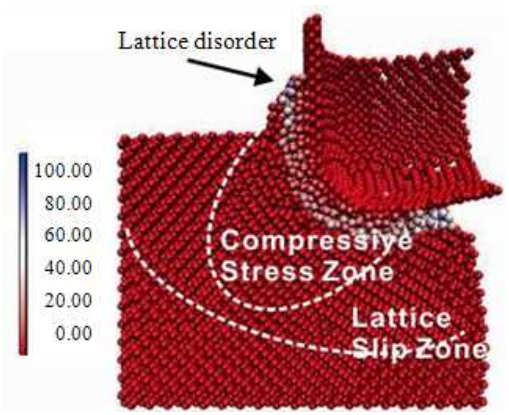

(a)

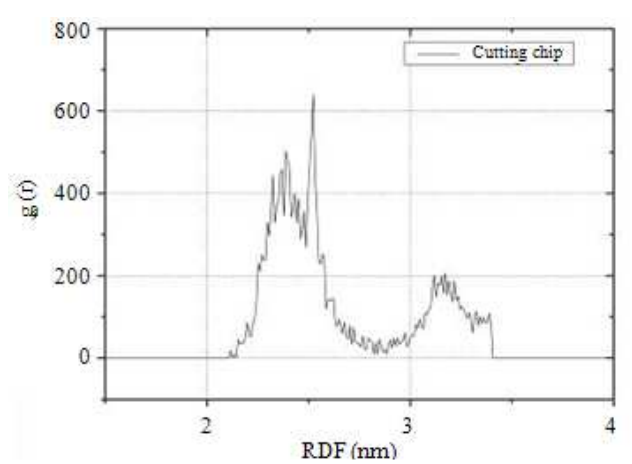

(b)

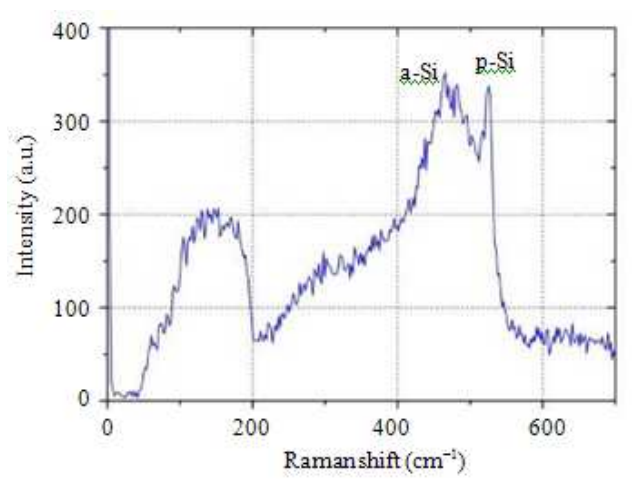

(c)

Fig. 4: Lattice disorder: (a) Schematic for lattice disorder; (b) RDF of cutting chip; (c) Raman spectrum of real cutting chip

Cutting force variation: Cutting forces with various tool cutting edges are simulated for different cutting depth, as shown in Fig. 5. It could be seen that when the cutting depth are below a certain value, all the cutting forces are homogeneous and without obvious increment, indicating no cutting chip formation. As the cutting depth above the critical value, the cutting forces start to increase, reveals the accumulation of cutting chips on the tool rake face. Figure 6 is the typical cutting force curves with and without chip formation. 


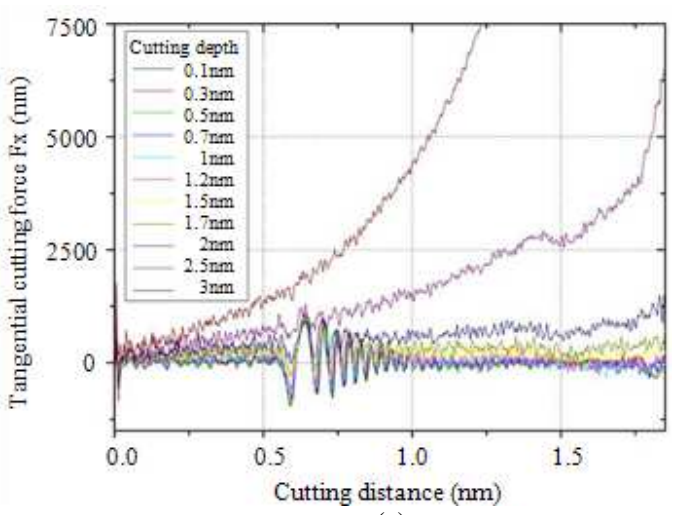

(a)

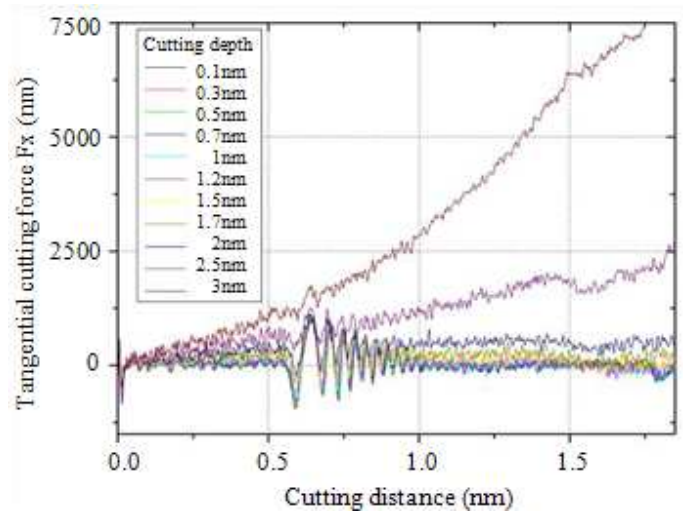

(c)

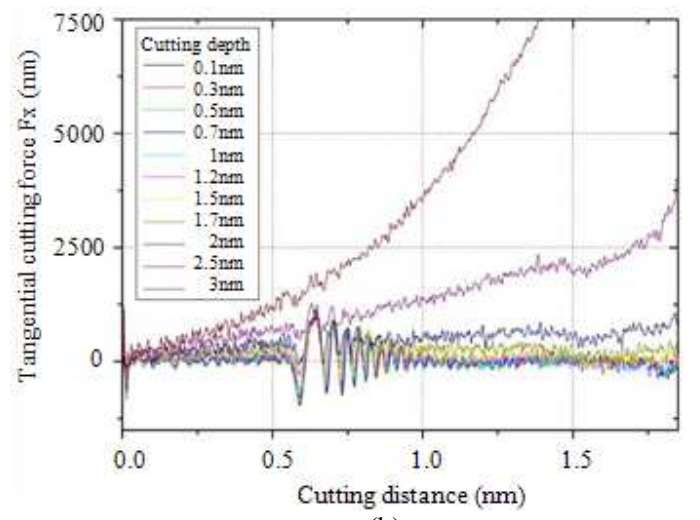

(b)

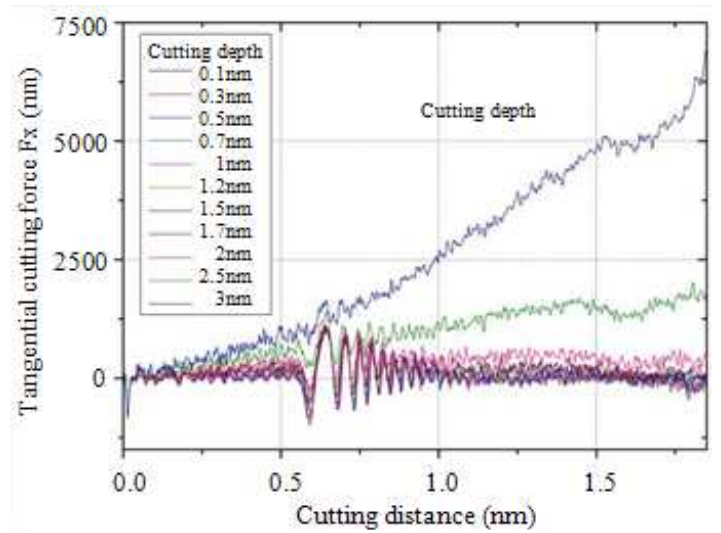

(d)

Fig. 5: Cutting forces with various cutting depths, (a) $1 \mathrm{~nm}$ cutting edge; (b) $1.5 \mathrm{~nm}$ cutting edge; (c) $2 \mathrm{~nm}$ cutting edge; (d) $2.5 \mathrm{~nm}$ cutting edge

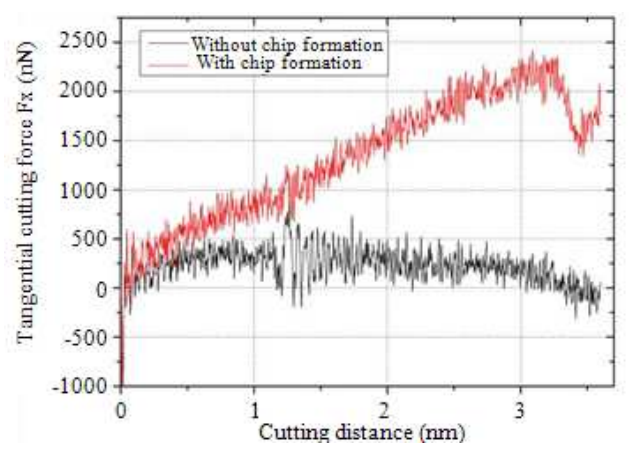

Fig. 6: Cutting force for chip formation

When the cutting depth is larger than chip formation threshold, cutting force increases gradually as the chips accumulate on the tool rake face and then exhibits a small decrease for chip removal. However, when the cutting depth is below the threshold, deformed silicon atoms could only flow along the cutting edge and the cutting force does not show obvious increment.

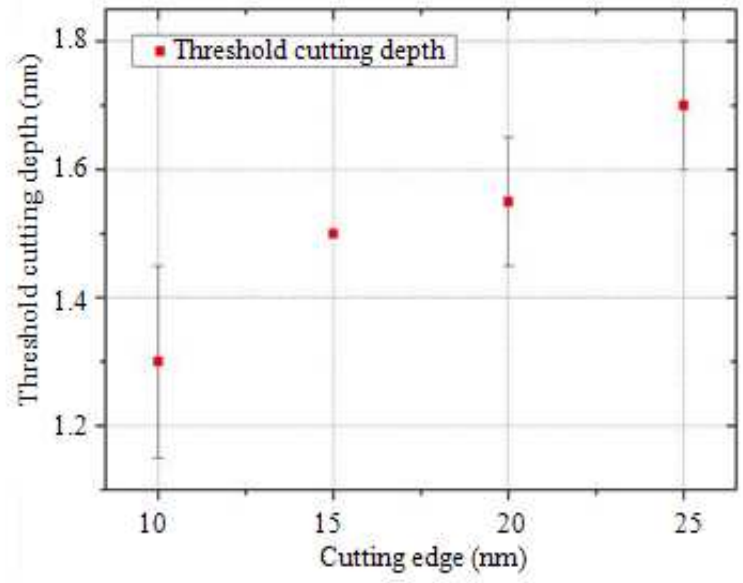

Fig. 7: Threshold cutting depth for chip formation

Figure 7 shows the chip formation threshold for different tool edges. The value increases in a small range as cutting edge value increase. 


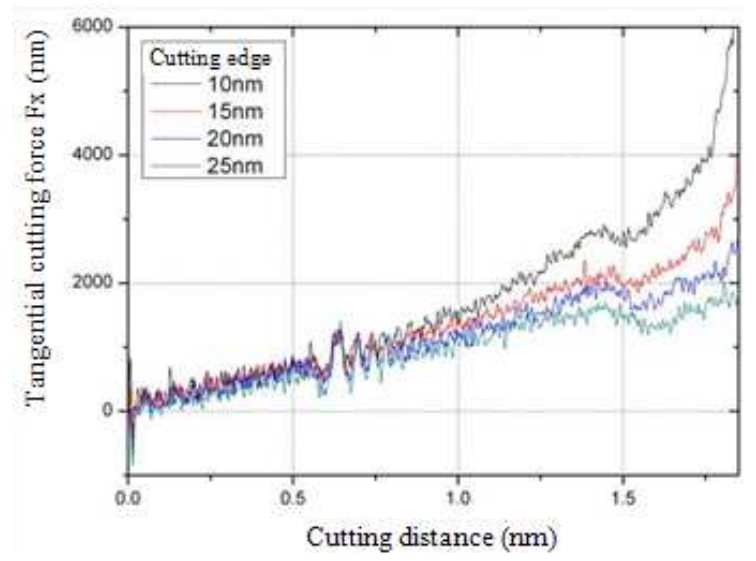

Fig. 8: Cutting force for different cutting edges

Figure 8 is the cutting force variation due to the change of the tool cutting edge. The cutting depth used is 2.5 $\mathrm{nm}$ which is above the chip formation threshold. Cutting forces decrease as the cutting edge value increase. As the threshold for cutting chip formation increase, the chip accumulation becomes smaller and the cutting force decrease accordingly.

\section{CONCLUSION}

Molecular dynamics simulations were carried out to investigate the cutting process and chip formation mechanism in nanometric cutting of monocrystalline silicon at the aspect of cutting force. The following conclusions can be drawn:

- Monocrystalline silicon experienced lattice slip and distortion due to the extrusion imposed hydrostatic pressure, which is in good agreement with the cutting force curves and is the main reason for tool wear and surface fracture

- The threshold cutting depth for chip formation is given for various tool cutting edges. When there are no cutting chips formation, cutting forces are homogeneous and without obvious increment. When there are cutting chips formed, cutting forces increase gradually due to the chips accumulation on the tool rake face

- Cutting forces decrease as the cutting edge value increase when cutting with same cutting depth

\section{ACKNOWLEDGMENT}

The study was supported by ' 111 ' project (B07014) by the State Administration of Foreign Experts Affairs and the Ministry of Education, National
High Technology Research and Development Program (863 Program) (No. 2009AA044305) and National Natural Science Foundation of China (No. 90923038).

\section{REFERENCES}

De Chiffrea, L., H. Kunzmannb, G.N. Peggsc and D.A. Lucca, 2003. Surfaces in precision engineering, microengineering and nanotechnology. CIRP Annals-Manuf. Technol., 52: 561-577. DOI: 10.1016/S0007-8506(07)60204-2

Evans, D.J., 1983. Nonequilibrium molecular dynamics via Gauss's principle of least constraint. Phys. Rev., 28: 1016-1021. DOI: 10.1103/PhysRevA.28.1016

Fang, F.Z. and V.C. Venkatesh, 1998. Diamond cutting of silicon with nanometric finish. CIRP Ann. Manuf. Technnol., 47: 45-49. DOI: 10.1016/S0007-8506(07)62782-6

Fang, F.Z., H. Wu, W. Zhou and X.T. Hu, 2007. A study on mechanism of nano-cutting single crystal silicon. J. Mater. Process. Technol., 184: 407-410. DOI: 10.1016/J.JMATPROTEC.2006.12.007

Lee, W.B., 1990. Prediction of microcutting force variation in ultra-precision machining. Precis. Eng., 12: 25-28. DOI: 10.1016/0141-6359(90)90005-J

Tsuzuki, H., P.S. Branicio and J.P. Rino, 2007. Structural characterization of deformed crystals by analysis of common atomic neighborhood. Comput. Phys. Commun., 177: 518-523. DOI: 10.1016/J.CPC.2007.05.018 